\title{
CPAP erhöht das Sterberisiko
}

Fragestellung: Kann eine nächtliche CPAP-Beatmung bei Patienten mit zentralem Schlafapnoesyndrom und Herzinsuffizienz die Prognose verbessern?

Hintergrund: Bis zu einem Drittel aller Patienten mit Herzinsuffizienz haben auch ein Schlafapnoesyndrom. Bisher wurde angenommen, dass dieses Krankheitsbild insbesondere durch die intermittierenden exzessiven Blutdruckanstiege für Patienten mit vaskulären Erkrankungen gefährlich ist. Daher wird für diese Gruppe routinemäßig eine nächtliche CPAP-Beatmung empfohlen. Die SERVE-HF-Studie untersuchte dieses Konzept als erste prospektive randomisierte Studie.

Patienten und Methodik: In die Studie wurden 1.325 Patienten mit links-ventrikulärer Ejektionsfraktion von $45 \%$ und kleiner aufgenommen. Einschlusskriterien war ein Apnoe-Hypopnoe-Index von 15 oder mehr Ereignissen pro Stunde und eine zentrale Ursache des Schlafapnoesyndroms. Die Hälfte der Patienten wurde mit einer nächtlichen CPAP-Therapie behandelt, die anderen erhielten ausschließlich eine medikamentöse Therapie. Der primäre Endpunkt waren Tod, lebensrettende kardiovaskuläre Interventionen oder eine ungeplante Krankenhausaufnahme wegen Verschlechterung der bestehenden Herzinsuffizienz.

Ergebnisse: Die Patienten waren im Mittel 70 Jahre alt und $90 \%$ waren übergewichtige Männer. Die mittlere linksventrikuläre Ejektionsfraktion betrug $32 \%$. Fast alle Patienten wurden mit ACE-Hemmern oder Angiotensin-RezeptorBlockern, Betablockern und Diuretika behandelt. Die Zahl hypopnoeischer Ereignisse pro Stunde betrug bei Baseline 32. Nach zwölf Monaten ergab sich kein signifikanter Unterschied in der Häufigkeit des primären Endpunkts mit 54,1\% in der CPAP-Gruppe und 50,8\% in der Kontrollgruppe. Es zeigte sich allerdings eine signifikant erhöhte Gesamtmortalität und kardiovaskuläre Sterblichkeit mit einer Risikoerhöhung von $28 \%$ bei Patienten mit

Cowie MR, Woehrle H, Wegscheider $\mathrm{K}$ et al. Adaptive servoventilation for central sleep apnea in systolic heart failure. N Engl J Med 2015; 368: 1613-24

\section{CPAP-Beatmung.}

Schlussfolgerungen: Bei Patienten mit einem Schlafapnoesyndrom und systolischer Herzinsuffizienz führt eine nächtliche CPAP-Beatmung zu einer erhöhten Sterblichkeit.

\section{- Kommentar von Hans-Christoph Diener, Essen}

\section{Nicht routinemäßig mit CPAP behandeln}

Die Ergebnisse dieser großen und gut durchgeführten randomisierten Studie stehen im Gegensatz zu einer Reihe von kleineren Studien und Metaanalysen, die alle eine Wirkung der nächtlichen CPAP-Beatmung gezeigt hatten. Dies ist erneut der Beweis, dass bestimmte Therapien nur dann in der Praxis eingesetzt werden sollen, wenn sie durch große randomisierte Studien in ihrer Wirkung belegt sind. Es könnte sein, dass das zentrale Schlafapnoesyndrom ein kompensatorischer Mechanismus bei Patienten mit schwerer Herzinsuffizienz ist. Das Schlafapnoesyndrom führt zu einer ausgeprägten sympathischen Aktivierung und zu einem Anstieg des Lungenvolumens. Es könnte auch sein, dass sich der positive Ventilationsdruck negativ auf die Herzfunktion auswirkt. Die Quintessenz dieser Studie ist, dass Patienten mit systolischer Herzinsuffizienz und Schlafapnoesyndrom nicht routinemäßig mit einer CPAP-Maske behandelt werden sollten. Allerdings sollte diese Therapie bei Patienten mit ausgeprägter Tagesmüdigkeit angeboten werden. 